\title{
$\angle S$ Research Square \\ Prevalence of Infection With Dirofilaria Immitis in Cats in Townsville, Australia
}

Carl Adagra ( $\square$ cadagra@hotmail.com )

James Cook University College of Medicine and Dentistry https://orcid.org/0000-0001-7120-6151

Richard Squires

James Cook University College of Public Health Medical and Veterinary Sciences

Angela Busst

Wulguru Veterinary Clinic

Jennifer Elliman

James Cook University College of Public Health Medical and Veterinary Sciences

Constantin Constantinoiu

James Cook University College of Public Health Medical and Veterinary Sciences

\section{Research}

Keywords: Dirofilaria immitis, Australia cats, PCR, DNA

Posted Date: July 20th, 2020

DOI: https://doi.org/10.21203/rs.3.rs-42351/v1

License: (c) (i) This work is licensed under a Creative Commons Attribution 4.0 International License.

Read Full License

Version of Record: A version of this preprint was published at Veterinary Parasitology: Regional Studies and Reports on April 1st, 2021. See the published version at https://doi.org/10.1016/j.vprsr.2021.100580. 


\section{Abstract \\ Objective}

Dirofilaria immitis commonly infects Australian dogs. Studies on the prevalence of infection by this parasitic helminth in Australia cats are rare and relatively old. Data obtained from other countries would suggest a likely prevalence of $4.7-16 \%$. The current study aimed to determine the prevalence of $D$. immitis in an endemic region of Australia by antigen, antibody and PCR testing.

\section{Methods}

172 healthy cats over 6 months of age from the Townsville region of Australia were tested for $D$. immitis specific antibodies and antigen using a commercially available kit. 50 samples were subsequently retested using a second commercially available antibody kit. 48 of these samples were checked for $D$. immitis DNA using PCR.

\section{Results}

No cat tested positive on any test. The Ausvet Epitools epidemiology calculator was used to calculate prevalences. Maximum antigen (1.27\%), antibody $(2.1 \%)$ and PCR $(2.1 \%)$ prevalences were calculated.

\section{Conclusion}

Our results suggest that the prevalence of heartworm infection in pet cats in this region of Australia is lower than expected based on data from other areas around the world.

\section{Introduction}

Dirofilariosis (heartworm disease, HWD) is common in dogs in many areas of the world, especially in wet tropical and subtropical regions ${ }^{1-8}$. Dirofilaria immitis is a mosquito-transmitted nematode that can cause severe cardio-pulmonary pathology and death in canids and other animals ${ }^{2,9,10}$. Cats can be infected but they are less suitable hosts than dogs. Less larvae develop into adults and adult worms have a shorter lifespan ${ }^{4}$. Unfortunately, because the pulmonary arteries in cats are smaller than in most dogs, infections with even a single worm can have fatal consequences and mortality rates are higher in infected cats than in dogs $s^{9,10}$.

Diagnosis of feline heartworm disease can be challenging. After infection, the arrival and death of worms within the lungs are thought to stimulate an acute injury. Clinical signs in cats may be absent or nonspecific such as loss of appetite, lethargy, coughing, dyspnoea and vomiting ${ }^{9}$. Early radiographic changes such as bronchointerstitial lung disease, lobar pulmonary arterial enlargement and pulmonary 
hyperinflation are also non-specific and often subtle ${ }^{11,12}$. This can lead to the disease being misdiagnosed as asthma or chronic bronchitis ${ }^{13}$. Serologic confirmation of feline heartworm disease is also problematic. Infections typically consist of low numbers of short-lived worms, often of a single sex ${ }^{6}$. As routine antigen tests detect what is thought to be a uterine antigen produced only by adult female worms over 5.6 months of age, they will not detect infection with juveniles or infections involving only male worms ${ }^{9,14}$. Another reported cause of false negative tests is the formation of antigen-antibody complexes that can 'shroud' parasite antigens and prevent their detection by the antigen test kit ${ }^{14}$. In dogs, presence of circulating microfilaria can be used to diagnose infections. However, in cats even if male and female adult worms are both present, microfilaria may be only transiently present in blood and are usually present in low numbers, if at all ${ }^{15}$. For these reasons, diagnosis of feline heartworm infections is difficult and often requires a combination of tests ${ }^{15}$.

The prevalence of infection with $D$. immitis in cats has been reported to be $5-20 \%$ of the concurrent prevalence in the local dog population ${ }^{2-10}$. However, there are several reasons why revisiting the prevalence of this worm in Australian cats is warranted. Studies on the prevalence of infection with $D$. immitis in cats in Australia are rare and relatively old ${ }^{16,17}$. Australian research indicates that heartworm infections in cats may be more prevalent than previously thought ${ }^{9}$. The prevalence of HWD in the world may also be changing due to the effects of climate change on the geographical range of mosquito vectors. It has also been reported that lack of efficacy of some heartworm preventative medications in dogs may potentially be increasing the pool of infected animals, that are then available to be bitten and transmit the disease to mosquitos in endemic areas ${ }^{18}$.

The prevalence of infection with D.immitis in cats from other endemic regions of the world has been studied. Based on detection using antibody tests, the prevalence was $4.7 \%$ in the Po valley of Northern Italy, $6.7 \%$ in Taiwan, $7.3 \%$ in Madrid, $11.47 \%$ in Barcelona, $16 \%$ in northern Italy and $17 \%$ in Florida. The prevalence using antigen tests are $0.2 \%$ in Madrid and $3.1 \%$ in Taiwan ${ }^{2-8}$.

PCR testing for detection of heartworm DNA has been available for more than a decade ${ }^{19-21}$. PCR is reported to have a very high sensitivity for $D$. immitis when microfilaria are present in the blood ${ }^{19-21}$. It was thought that including this test could reveal more information about the rate of feline heartworm infection.

\section{Materials And Methods}

172 blood samples were collected over a 2 year period. Samples were only collected from cats that were over 6 months of age, where blood was being collected for unrelated reasons (usually routine health screens or testing for Feline Immunodeficiency Virus). Blood was collected into EDTA and plain tubes. Plasma from the EDTA tubes was separated by centrifugation antigen/antibody testing. 152 of these samples were collected from cats from private households where information was available on the cat's age, heartworm prophylaxis status and access to the outdoors. None of the cats had clinical signs 
suggestive of heartworm infection. Of the 152 cats living in private households, $53.9 \%$ were male. $63.9 \%$ had access to the outdoors. $16.4 \%$ of cats were on adequate heartworm preventative medications which was defined as monthly usage of products containing milbemycin oxime, selemectin or imidacloprid and moxidectin. The mean age of cats tested was 9.8 years.

20 samples were taken from cats housed in an animal rescue facility. For these cats information about age and prior history was not available. These cats had been examined by a veterinarian, considered to be healthy, and were all estimated to be over 6 months of age.

Initial testing consisted of heartworm antigen and antibody testing using the Bionote Anigen Rapid Feline Heartworm Antigen/Antibody Test Kit (Bionote Inc. Seoul, Korea), following the manufacturers instructions. 50 random samples were subsequently retested for heartworm antibodies using Heska Solo Step antibody test kits(Heska Corporation. Loveland, United States), using the protocol provided with the kits.

PCR testing was carried out on 48 randomly selected whole blood samples using previously reported primers for Dirofilaria mitochondrial cytochrome oxidase gene, subunit $\mathrm{I}^{21}$. After optimisation of the nucleic acid extraction and PCR protocols using whole blood from dogs confirmed to be infected using microscopy, 100 ul of whole blood was diluted with 100 ul PBS, prior to extraction of genomic DNA using a QIAmp blood mini kit (Qiagen, Hildon, Germany) with elution into 200 ul AE buffer. DNA extracted from isolated $D$. immitis was used as a positive control. The reaction mixture for the real time PCR was made up using a Bioline Sensifast SYBR No-ROX mastermix in a total volume of 20 ul. The reaction mix containing $10 \mathrm{ul} \mathrm{mastermix,0.3uM} \mathrm{of} \mathrm{each} \mathrm{primer} \mathrm{(COXdirHRMF:} \mathrm{5'-AGTATGTTTGTTTGAACTTC-} \mathrm{3',}$ COXdirHRMR: 5'-AACGATCCTTATCAGTCAA- $3^{\prime}$ ) and 2.5 ul of DNA. PCR was carried out on a Qiagen Rotogene 6000 real time PCR machine using the following conditions; $95^{\circ} \mathrm{C}, 3 \mathrm{~min}$, followed by 40 cycles of $95^{\circ} \mathrm{C}, 30 \mathrm{~s}, 52^{\circ} \mathrm{C}, 15 \mathrm{~s}, 72^{\circ} \mathrm{C}, 30 \mathrm{~s}$ acquiring to the green channel. This was followed by a melt step using a ramp from $72{ }^{\circ} \mathrm{C}$ to $95^{\circ} \mathrm{C}$ with a Hold for $90 \mathrm{~s}$ on the 1 st step and holds for $5 \mathrm{~s}$ on next steps.

\section{Results}

No cats tested positive by any of the tests used.

Antigen / Antibody Detection Tests

Positive control lines were present in all kits used.

\section{PCR}

While many samples contained amplification products, examination of the melt curve identified a peak for the positive control at $80.5^{\circ} \mathrm{C}$ while all sample positive peaks were at $85.5^{\circ} \mathrm{C}$. Sequencing confirmed the $D$. immitis positive control peak to be $D$. immitis and the sample peak at $85.5^{\circ} \mathrm{C}$ to be feline genomic DNA. 


\section{Data analysis}

The Ausvet Epitools epidemiology calculator (https://epitools.ausvet.com.au/) was used to calculate maximum actual prevalence of $D$. immitis with upper $95 \%$ confidence limits using the following data:

Maximum antigen prevalence using the Bionote Anigen Rapid kit.

Sensitivity was specified as $94.1 \%$ based on data provided by the manufacturer. Specificity was estimated as $99 \%$ based on the lack of any positive samples. The maximum actual prevalence was calculated at $1.27 \%$.

Maximum antibody prevalence using the Bionote Anigen Rapid kit.

Sensitivity and specificity data were not available for the antibody component of the test therefore a different approach was used to calculate maximum antibody prevalence based on 172 negative samples. The Townsville cat population was estimated at 50,000 based on data available from Pet Ownership in Australia, Animal Medicines Australia, the 2016 census and an estimation of the number of stray animals. Using the equation:

$$
a=1-\left(1-\frac{n}{N-\frac{e-1}{2}}\right)^{e}
$$

Where

$\mathrm{CL}=$ The level of confidence.

$\mathrm{n}=$ The number of individuals in the sample.

$\mathrm{N}=$ The number of individuals in the population.

$\mathrm{e}=$ The number of individuals presenting the event in the population that can be detected.

The maximum antibody prevalence was calculated to be less than $13 \%$ with a $95 \%$ confidence limit.

Maximum antibody prevalence using the Heska Solo-Step kit

Sensitivity was specified as $96 \%$ and specificity as $98 \%$ based on data provided by the manufacturer. The maximum antibody prevalence was calculated to be less than $2.1 \%$. 
Sensitivity and specificity of this specific PCR assay is undetermined, but based on prior data a conservative estimate would be $98 \%$ for both sensitivity and specificity. Using this data the maximum PCR prevalence would be $2.1 \%$.

\section{Discussion}

The low prevalence of $D$. immitis infections in cats in this region was unexpected, but supported by a very low rate of clinically reported feline heartworm cases (Adagra, personal observations). The prevalence of $D$. immitis infection in cats in a particular geographic location is typically much lower than the prevalence in dogs, but the differences are quite variable by region ${ }^{2-6}$. This variability is thought to depend on the variable willingness of local mosquito species to feed on cats versus dogs ${ }^{2-9}$. When the mosquito species that are considered to be heartworm vectors in the Townsville region are compared to the mosquito species that are identified to be natural hosts of $D$. immitis in Florida in the United States, only one is endemic to both regions; Culex quinquefasciatus $s^{22-24}$. This species is thought to have a preference to feed on poultry rather than humans ${ }^{23}$ and it is quite possible that other feeding preferences exist. However, there are over 300 species of mosquito present in Australia ${ }^{23}$.The role of different species of mosquito as vectors in HWD in Australia has not been adequately studied and is also liable to change over time.

Whilst climate change is likely to alter population densities of mosquitos worldwide, human manipulation of mosquito populations may also alter heartworm prevalences. Recently there was a planned release of large numbers of Wolbachia pipientis-infected Aedes aegypti mosquitos in Townsville ${ }^{25}$. This successfully established endemic Wolbachia infection into the local $A$. aegypti population. This led to a marked decrease in human cases of Dengue fever ${ }^{25}$. Any effect on heartworm prevalence from such interventions is unknown. Wolbachia is an important symbiote for $D$. immitis, which is usually transmitted vertically ${ }^{26}$. Whether Wolbachia presence within the mosquitos could aid transmission of $D$. immitis or possibly enable the worm to use atypical mosquito species as vectors is yet to be researched.

We have estimated the maximum antibody prevalence in cats in this region to be $2.1 \%$ and the maximum antigen and PCR prevalence rates to be $1.27 \%$ and $2.1 \%$. The true rates could be lower than this. Not having a single positive sample raises questions about how low the true prevalence is, as well as the validity of the diagnostic tests being used. Ideally we would have continued testing cats until we obtained at least a single positive sample to address these concerns but were unable to do this due to financial constraints. The manufacturers of the commercial kits were able to provide data supporting their validity. We did attempt independently to validate the kits by contacting four feline specialist centres across eastern Australia to obtain blood from a $D$. immitis infected cat. None had seen a cat that had tested positive to heartworm infection in recent years which, in itself supports our low prevalence findings.

Maximum antigen prevalence in this study was calculated at $1.27 \%$. Antigen testing for $D$. immitis in cats often produces false negative results leading to an artificially low maximum prevalence ${ }^{14}$. Whilst the 
specificity of commercial test kits is good, false negatives can be obtained from infections with juvenile worms(less than 5.6 months old), infections where only male worms are present, if antigen-antibody binding occurs and possibly after the administration of heartworm preventatives or doxycycline ${ }^{14}$.

The phenomenon of antibody-antigen binding has been studied in dogs ${ }^{14}$. Heat treatment of sera increases the sensitivity of antigen detection test kits and can resolve discordant results in dogs that are microfilaria positive- but antigen negative ${ }^{14}$. We opted not to perform heat treatment on our samples. Heat treatment has been reported to increase the number of false positive results in one study ${ }^{27}$ and was not shown to increase the number of positive feline heartworm antigen test results in a second study ${ }^{6}$. Theoretically increasing the sensitivity of our test from $94.1-99 \%$ would have decreased the calculated maximum true prevalence only from $1.27-1.21 \%$. However if heat treating sera generated a single false positive, this would have significantly increased our calculated maximum true prevalence from 1.27$2.22 \%$. The use of concurrent PCR testing was intended to help reveal more about any false-negative results and confirm the species involved, of course no relevant data were obtained.

Climate across the world is usually classified by the Köppen-Geiger climate classification system which assigns climate types a code based on seasonal precipitation and temperature patterns ${ }^{28}$. The climate of the Townsville region is classified Aw (Tropical savanna climate with dry-winter characteristics) ${ }^{29}$. Most regions where heartworm prevalence in cats has been studied are classified Csa or Cfa (Mediterranean hot summer climate or humid subtropical climate) ${ }^{29}$. However, Florida (USA) is a location where a large zone of the Aw climate type can be found, as well as Cfa, Am (Tropical monsoon climate) and Af (Tropical rainforest climate). In a recent study of HWD prevalence in Florida, animals were recruited from three locations ${ }^{6}$. One of these was Miami-Dade county where Aw,Am and Af climate types are found. The prevalence of HWD was not significantly different in this county to the more northern locations studied where only Cfa climate is found.(Jessica Rodriguez, Personal communication). Miami-Dade county is much wetter than the Townsville region ${ }^{30,31}$ and we considered the possibility that sparse winter rainfall could be reducing the number of mosquito vectors and therefore the overall heartworm prevalence in this region. However, the prevalence of $D$. immitis in dogs on entry to a local rescue shelter was determined by conventional antigen testing to be $22.1 \%$ (Constantinoiu C. and Coleman, G., personal communication). This canine HWD rate in the Townsville region would suggest that localised climate conditions are not the cause for the lack of feline cases in this study.

Our sample population consisted primarily of pet cats. In Florida in 2019, 100 cats from animal shelters were tested for heartworm antibody, $17 \%$ were positive ${ }^{6}$. However in the same location and year, the results of 32,067 feline heartworm antibody tests were reported to the Companion Animal Parasite Council. The overall positive rate was $0.45 \%$ ( 1 in 225 cats $)^{32}$. The heartworm prophylaxis status of the cats on which the 32,067 tests were performed is unknown, but presumably the data would be skewed towards cats that were considered to be on inadequate heartworm prophylaxis. This would suggest that maybe another factor such as poor nutrition or other parasite burden leads stray cats to be more 
susceptible to developing heartworm antibodies and presumably disease. Of course, we would have required a much larger sample size to detect a prevalence this low.

The current research has been limited by sample numbers and our lack of positive test results. This makes it difficult to be confident when calculating the prevalence of HWD. However, the prevalence of $D$. immitis infection in cats in this study is much lower than expected considering the prevalence in dogs from this region. The authors still support the routine use of heartworm prophylaxis in Australian cats. The consequences of infection in this species are severe, if not fatal, with occasional cases still being reported in the country.

\section{Declarations}

Ethics approval and consent to participate.

Advice was sought from the James Cook University Ethics Approval Committee by the authors. We were advised ethics approval was not required as "waste" blood was used.

Consent for publication

The Authors consent to the publication of this article.

Availability of data and material

Data is available after correspondence with the Authors.

Competing interests

The Authors do not have any competing interests to declare.

Funding

This research was funded by James Cook University and Tropical Qld Cat Clinic Pty Ltd (Owned by Carl Adagra).

Authors contributions

All authors contributed to the manuscript.

Acknowledgements

The Authors would like to acknowledge Glen Coleman and Jessica Rodriguez who assisted with data on this project.

\section{References}


1. Nguyen C, Koh WL, Casteriano A, et al. Mosquito-borne heartworm Dirofilaria immitis in dogs from Australia. Parasite Vector. 2016;9:535.

2. Montoya-Alonso JA, Morchón R, Falcón-Cordón Y, et al. Prevalence of heartworm in dogs and cats of Madrid, Spain. Parasite Vector. 2017;10:354-6.

3. Lu TL, Wong JY, Tan TL, et al. Prevalence and epidemiology of canine and feline heartworm infection in Taiwan. Parasite Vector. 2017;10:484-5.

4. Kramer L, Genchi C. Feline heartworm infection: serological survey of asymptomatic cats living in northern Italy. Vet Parasitol. 2002;1:43-50.

5. Carleton RE, Tolbert MK. Prevalence of Dirofliaria immitis and gastrointestinal helminths in cats euthanised at animal control agencies in northwest Georgia. Veterinary Parisitology. 2004;119:31926.

6. Hays KM, Rodriguez JY, Little SE, et al. Heartworm prevalence in dogs versus cats: Multiple diagnostic modalities provide new insights, Veterinary Parasitology, 2020;109142.

7. Montoya-Alonso JA, Carreton E, Garcia-Gausch L, et al. First epidemiological report of feline heartworm infection in the Barcelona metropolitan area. Parasite Vector. 2014;7:506.

8. Park H, Lee S, Lee W, et al. Prevalence of Dirofilaria immitis infection in stray cats by nested PCR in Korea. Korean J Parasitol. 2014;52:691-4.

9. Litster AL, Atwell RB. Feline heartworm disease: a clinical review. J Feline Med Surg. 2008;10:13744.

10. Lee A, Atkins CE. Understanding feline heartworm infection: Disease, diagnosis and treatment. Top Companion Anim M. 2010;25:224-30.

11. Venco L, Genchi C, Genchi M. Clinical evolution and radiographic findings of feline heartworm infection in asytmptomatic cats. Vet Parisitol. 2008;158:232-7.

12. Selcer BA, Newell SM, Mansour AE, et al. Radiographic and 2-D Echocardiographic findings in eighteen cats experimentally exposed to D. immitis via mosquito bites. Vet Radiol Ultrasoun. 1996;37:37-44.

13. McCall JW, Genchi C, Kramer LH. Advances in Parasitology Chapter 4. 2008;66:195-250.

14. Little $S$, Saleh M, Wohltjen $M$, et al. Prime detection of Dirofilaria immitis: understanding the influence of blocked antigen on heartworm test performance. Parasit Vectors. 2018;11:186.

15. Cote E, MacDonald KA, Meurs KM, et al. Feline Cardiology Chapter. 2011;23:353-61.

16. Kendall K, Collins GH, Pope SE. Dirofilaria immitis in cats from inner. Sydney Aust Vet J. 1991;68:356-7.

17. Holmes PR, Kelly JD. The incidence of Dirofilaria immitis and Dipetalonema reconditum in dogs and cats in Sydney. 1973;49:55.

18. Bourguinat C, Lee ACY, Lizundia R, et al. Macrocyclic lactone resistance in Dirofilaria immitis: Failure of heartworm preventives and investigation of genetic markers for resistance. Vet Parasitol. 2015;210:167-78. 
19. Gioia G, Lecová L, Genchi M, et al. Highly sensitive multiplex PCR for simultaneous detection and discrimination of Dirofilaria immitis and Dirofilaria repens in canine peripheral blood. Vet Parasitol. 2010;172:160-3.

20. Thanchomnang T, Intapan PM, Lulitanond V, et al. Rapid detection of Dirofilaria immitis in mosquito vectors and dogs using a real-time fluorescence resonance energy transfer PCR and melting curve analysis. Vet Parasitol. 2010;168:255-60.

21. Albonico F, Loiacono M, Gioia G, et al. Rapid differentiation of Dirofilaria immitis and Dirofilaria repens in canine peripheral blood by real-time PCR coupled to high resolution melting analysis. Vet Parasitol. 2014;200:128-32.

22. Mosquitoes. in Townsville factsheet. www.eliminatedengue.com. Accessed 27th June 2020.

23. Mosquitoes of Australia website. University of Sydney, Department of Medical Entymology. http://medent.usyd.edu.au/photos/mosquitoesofaustralia.htm Accessed 27th June 2020.

24. Nayar JK, Connelly CR. Mosquito-Borne Dog Heartworm Disease 2017. http://edis.ifas.ufl.edu. Accessed 27th June 2020.

25. O'Neill SL, Ryan PA, Turley AP, et al Scaled deployment of Wolbachia to protect the community from Aedes transmitted arboviruses. Gates Open Res F1000 Research, Ltd., 2018;2:36.

26. McHaffie J. Dirofilaria immitis and Wolbachia pipientis: A thorough investigation of the symbiosis responsible for canine heartworm disease. Parasitol Res 2012:499-502.

27. Venco L, Manzocchi S, Genchi, et al. Heat treatment and false positive heartworm antigen testing in ex vivo parasites and dogs naturally infected by Dirofilaria repens and Angiostrongylus vasorum. Parasit Vectors. 2017;10:476.

28. Bacon S, Aebi A, Calanca P, et al. Quarantine arthropod invasions in Europe: The role of climate, hosts and propagule pressure. Divers Distrib. 2014;20:84-94.

29. Kottek M, Grieser J, Beck C, et al. World Map of the Köppen-Geiger climate classification updated. Meteorol. Z. 2006;15:259-263. http://koeppen-geiger.vu-wien.ac.at/present.htm Accessed 27th June 2020.

30. US Climate data website. https://www.usclimatedata.com/climate/miami/florida/unitedstates/usfl0316.

31. Accessed. 27th June 2020.

32. Australian Government Bureau of Meteorology. http://www.bom.gov.au/qld/townsville/climate_Townsville.shtml Accessed 27th June 2020.

33. Companion Animal Parasite Council website. https://capcvet.org/maps/\#2019/all/feline-heartwormab/cat/united-states/florida/. 